\title{
Selección genética de plantas elites de palma aceitera, utilizando software SELEGEN REML/BLUP
}

\section{Genetic selection of elite plants of oil palm using SELEGEN REML / BLUP software}

\author{
Carlos Oliva $^{1, *} ;$ Julián Chura Ch. $^{2}$; Harvey Pinedo ${ }^{3}$ \\ ${ }^{1}$ Phi Peruvian Consultores SAC, Av. Ricardo Palma $N^{\circ} 776$ - Urb. San Joaquín-Bellavista - Callao, Lima, Perú. \\ ${ }^{2}$ Universidad Nacional Agraria La Molina, Av. La Molina s/n - La Molina - Lima, Perú. \\ ${ }_{3}^{3}$ Palmagro SAC, Calle $2 \mathrm{Mz}$ E Lote 6 - Urb. El Bosque - Yarinacocha-Ucayali, Perú.
}

Recibido 14 mayo 2014. Aceptado 20 septiembre 2014.

\begin{abstract}
Resumen
La palma aceitera es uno de cultivos de la Amazonía Peruana, que genera en los inversionistas mayor interés, que ha permitido instalar al menos 70 mil ha. Al Perú ha ingresado semillas de palma aceitera de alto valor genético, por su resistencia a enfermedad, plagas y alto rendimiento, pero durante el tiempo ha experimentado variabilidad en los diferentes ecosistemas del país. Este trabajo tuvo por objetivo la selección genética computarizada para la selección de plantas elites de alto rendimiento de racimos de fruta fresca (RFF) de palma aceitera. Para realizar el análisis genético computarizado, se dispuso del software SELEGEN Rml/Blup, que es un programa diseñado para el análisis y la selección. Benin y Costa de Marfil son las que tienen mejor promedio, cuyo valor mínimo es de $22,1 \mathrm{~kg} / \mathrm{planta}$ y el valor máximo corresponde de $375,9 \mathrm{~kg} / \mathrm{planta}$. El hibrido 2301 tiene los mejores promedios de rendimiento, seguido por el híbrido 2401, cuyo valores de rendimiento extremos máximo superior a $340 \mathrm{~kg} /$ planta. El primer año, el rendimiento promedio fue de 46,62 $\mathrm{kg} /$ planta y para el tercer año de producción el promedio pasó a $142,82 \mathrm{~kg} / \mathrm{pl}$. La repetibilidad individual para rendimiento de RFF kg/planta en ambos grupos 2007 y 2008 fue de 0,10 y la repetibilidad del promedio de cosechas fue de 0,87 y 0,82 para los grupos 2007 y 2008, respectivamente. Esto propició una exactitud selectiva de 0,93 para el grupo 2007 y de 0,90 para el grupo del 2008.
\end{abstract}

Palabras clave: SELEGEN, plantas elites, rendimiento, selección.

\begin{abstract}
Oil palm cultivation is one of the Peruvian Amazon, which generates more interest among investors, which has allowed to install at least 70 thousand of ha. When Peru has entered oil palm seeds of high genetic value, resistance to disease, pests and high performance, but over time has experienced variability in different ecosystems of the country. This study aimed to computerized genetic selection for selection of elite plants of high performance fresh fruit bunches (FFB) of oil palm. For the computerized genetic analysis were available from SELEGEN Rml/Bloop software program that is designed for the analysis and selection. Benin and Ivory Coast are the ones with the best average, the minimum value is $22.1 \mathrm{~kg} / \mathrm{plant}$ and the maximum value corresponds to $375.9 \mathrm{~kg} /$ plant. The 2301 hybrid has the best average performance, followed by the hybrid 2401 , the maximum yield extreme values exceeding $340 \mathrm{~kg} / \mathrm{plant}$. The first year, the average yield was 46.62 $\mathrm{kg} / \mathrm{plant}$ and for the third year of production, the average rose to $142.82 \mathrm{~kg} / \mathrm{pl}$. Individual performance repeatability for RFF kg/plant in both groups 2007 and 2008 was 0.10 and the repeatability of the average crop was 0.87 and 0.82 for groups 2007 and 2008, respectively. This led to a selective accuracy of 0.93 for 2007 and 0.90 for the group 2008 .
\end{abstract}

Keywords: SELEGEN, elite plants, performance, selection.

\section{Introducción}

El cultivo de palma aceitera, es una de las actividades más importantes de la Amazonía Peruana, acción que ha

\footnotetext{
* Autor para correspondencia

E-mail: olivaproyectos@gmail.com (C. Oliva).
} 
permitido instalar aproximadamente $70 \mathrm{mil}$ ha en Ucayali (Gobierno regional de Ucayali, 2011), las mismas que están en diferentes ecosistemas y en diferentes edades. Se estima que en el año 2012 al Perú ha ingresado 10 millones de semillas de palma aceitera, procedentes de 5 países como: Benín, Costa de Marfil, Costa Rica, Ecuador y Colombia.

Las semillas que ingresaron a nuestro país son mejoradas, las mismas que presentan solidas fichas técnicas que proyectan alto rendimiento $(60 \mathrm{~kg} /$ planta al primer año de producción), por lo que ha generado altas expectativas en nuestro país. Después de algunos años en producción se ha podido observar que este material genético, viene experimentando altos niveles de variabilidad en el rendimiento (valores que fluctúan entre 15 a $160 \mathrm{~kg} /$ planta), esto debido al efecto de la adaptación del material genético bajo nuestras condiciones, creando incertidumbre y dificultad para elegir la semilla de mejor procedencia para implementar la frontera agrícola.

La obtención de semilla mejorada de palma aceitera es muy especializada, por lo que si no aprovechamos el material genético que ya está adaptado a nuestras condiciones y de alto rendimiento para obtener semillas de palma aceitera, seguiremos condicionados a la importación de semillas y por ende con la alta variabilidad en la producción. Por ello se hace necesarios tomar como base el material genético de las 5 procedencias e identificar fenotípicamente plantas de alto rendimiento y sobre esa data seleccionar plantas elites mediante "Selección Genética Computarizada RELM/BLUP".

Existen trabajos de selección genética computarizada realizada en especies perennes en nuestro país como el cacao y el camu camu cuyo análisis ha permitido ganancias genéticas encima del 100\%, permitiendo disponer de plantas elites de alto rendimiento como fuente de semillas para la ampliación de la frontera agrícola (Oliva y Resende, 2008; Oliva et al., 2014).

\section{Materiales y métodos}

Material genético

El material genético utilizado en la selección genética computarizada de palma aceitera de 5 procedencias (países) son: Benín 2301 (Cirad), Benín 2401 (Cirad), Costa de Marfil (Cirad), Costa Rica (ASD), Ecuador (Cirad), Colombia 1001 (Cirad). El material genético fue sembrado en campo definitivo en los años 2007 y 2008, por lo que fue posible evaluar plantas en producción.

\section{Variables de selección}

El análisis de selección genética computarizada, se realizó en base al rendimiento de racimos de fruta fresca (RFF) de palma aceitera, el mismo que fue dividido en 2 grupos (Grupo 01: Plantas procedentes de BENIN y Costa de Marfil; Grupo 02: Plantas procedentes de Costa Rica, Ecuador y Colombia), debido a diferencia de edad de las plantas y que por criterio técnico era absolutamente imposible analizarlos en un solo grupo.

Modelo genético

Pedrozo et al. (2009) proyectó el procedimiento del Modelo 63, modelo básico sin repetibilidad. Este modelo se utilizó cuando los datos repetidos eran tomados de plantas individuales sin el uso de diseños experimentales.

Del modelo estadístico $y=X m+W p+e$, donde " $y$ " es el vector de datos, " $m$ " es el vector de los efectos de la medición de (que se supone fijo) añadido a la media global, " $p$ " es el vector de los efectos permanentes de las plantas (+ efectos genotípica de los efectos ambientales permanentes) (supone que aleatorio) " $e$ " es el vector de errores o residuos (al azar).

Los valores genotípicos de cada planta serán estimados por $\hat{g}_{0}=M G+\beta_{p}\left(M P_{i}-\right.$ $M G)$, en que: $\beta_{p}=m \dot{p} / 1+(m-1), \dot{p}$ : coeficiente de determinación del valor fenotípico permanente o repetitividad de la media de: $m=4$ mediciones en el individuo. $\dot{p}$ : Estimativa de la repetitividad individual. $M G$ : media general de las plantas en varias mediciones; $M P i$ : media 
general da la planta $i$ en las varias mediciones.

La ganancia genética será estimada como el promedio de valores genotípicos de los individuos seleccionados.

\section{Resultados y discusión}

De las 5 procedencias, los de Benín y Costa de Marfil son las que tienen mejor promedio de peso de racimos por planta, debido a ello se explica el amplio rango de los valores extremos, cuyo valor mínimo es de $22,1 \mathrm{~kg} /$ planta y el valor máximo corresponde a $375,9 \mathrm{~kg} /$ planta. Para el caso de las procedencias Costa Rica, Ecuador y Colombia se observan que el promedio es menor a las procedencias de Benín y Costa de Marfil con 69,82 kg/planta, 43,30 $\mathrm{kg} /$ planta y $53,67 \mathrm{~kg} /$ planta respectivamente, esto debido a que los datos corresponden a los 2 primeros años de producción, pero a pesar de ello en la procedencia de Ecuador se observa el menor promedio con 43,302 $\mathrm{kg} /$ planta (Resende y Oliveira, 1997).

\section{Tabla 1}

Rendimiento de palma aceitera en el Perú según procedencias (en RFF kg/planta)

\begin{tabular}{ccccc}
\hline & \multicolumn{5}{c}{ Promedio Desviación } & & \\
Procedencia & kg/planta) & estándar & Mínimo & Máximo \\
\hline Benín & 98,03 & 54,67 & 22,1 & 375,9 \\
Costa de marfil & 92,38 & 43,41 & 26,0 & 216,6 \\
Costa rica & 69,82 & 35,69 & 33,5 & 172,8 \\
Ecuador & 43,30 & 8,68 & 24,0 & 59,7 \\
Colombia & 53,67 & 17,46 & 32,3 & 104,4 \\
Total & 85,47 & 49,45 & 22,1 & 375,9 \\
\hline
\end{tabular}

Dentro de las 5 procedencias, se consolidaron 6 híbridos, de código 2301, 2401, CIRAD, ASD, CIRAD (Ecuador) y 1001. Se observó que el hibrido 2301 tiene los mejores promedios de rendimiento, seguido por el híbrido 2401, ambos de procedencia BENIN. Además en ambos híbridos se observan valores de rendimiento extremos máximo superior a $340 \mathrm{~kg} /$ planta.

En los híbridos restantes ASD, CIRAD-E y 1001, que corresponden a las procedencias de Costa Rica, Ecuador y Colombia, se observó que la procedencia de Ecuador es el que reporta menor rendimiento promedio $(43,302 \mathrm{~kg} /$ planta $)$.

\section{Tabla 2}

Rendimiento de palma aceitera introducidas al Perú según híbridos (en RFF kg/planta)

\begin{tabular}{ccccc}
\hline Híbrido & $\begin{array}{c}\text { Promedio } \\
(\mathrm{kg} / \text { planta) }\end{array}$ & $\begin{array}{c}\text { Desviación } \\
\text { estándar }\end{array}$ & Mínimo & Máximo \\
\hline 2301 & 102,84 & 65,05 & 34,8 & 375,9 \\
2401 & 95,63 & 48,68 & 22,1 & 342,0 \\
CIRAD & 92,38 & 43,41 & 26,0 & 342,0 \\
ASD & 69,82 & 35,69 & 33,5 & 172,8 \\
CIRAD-E & 43,30 & 8,68 & 24,0 & 59,7 \\
1001 & 53,67 & 17,46 & 32,3 & 104,4 \\
Total & 85,47 & 49,45 & 22,1 & 375,9 \\
\hline
\end{tabular}

Para el grupo 2007, se encontró que el promedio de rendimiento de un año a otro es significativo, es decir, en el primer año de producción el rendimiento promedio fue de 46,62 kg/planta, con valores extremos que van desde 22,1 hasta $100 \mathrm{~kg} /$ planta. Para el segundo año de producción el promedio se incrementó a $100 \mathrm{~kg} /$ planta, con valores extremos de 71,3 hasta 133,4 $\mathrm{kg} /$ planta y para el tercer año de producción el promedio pasó a 142,82 $\mathrm{kg} /$ planta, con valores extremos que va desde 57,4 hasta 375,9 kg/planta (Tabla 3).

\section{Tabla 3}

Rendimiento de palma aceitera según evaluaciones en campo definitivo-Grupo 2007 (en RFF kg/planta)

\begin{tabular}{ccccc}
\hline Evaluación & $\begin{array}{c}\text { Promedio } \\
(\mathrm{kg} / \text { planta) }\end{array}$ & $\begin{array}{c}\text { Desviación } \\
\text { estándar }\end{array}$ & Mínimo & Máximo \\
\hline 1 & 46,62 & 8,48 & 22,1 & 100,9 \\
2 & 100,41 & 14,68 & 71,3 & 133,4 \\
3 & 142,83 & 56,64 & 57,4 & 375,9 \\
Total & 96,62 & 52,08 & 22,1 & 375,9 \\
\hline
\end{tabular}

Para el grupo 2008, se observaron diferencias marcadas en el rendimiento de un año para otro, por ello es fundamental la comparación de plantas que tengan similar edad y bajo condiciones de manejo uniformes. En el primer año el rendimiento promedio fue de $40,50 \mathrm{~kg} / \mathrm{planta}$, con valores extremos que van desde 32,3 hasta $51 \mathrm{~kg} / \mathrm{planta} \mathrm{y}$ en el segundo año el promedio se incrementó hasta 73,09 $\mathrm{kg} /$ planta con valores extremos que van desde $24 \mathrm{~kg} /$ planta hasta 172,8 kg/planta (Tabla 4). 


\section{Tabla 4}

Rendimiento de palma aceitera según evaluaciones en campo definitivo-Grupo 2008 (en RFF kg/planta)

\begin{tabular}{ccccc}
\hline Evaluación & $\begin{array}{c}\text { Promedio } \\
(\mathrm{kg} / \text { planta) }\end{array}$ & $\begin{array}{c}\text { Desviación } \\
\text { estándar }\end{array}$ & Mínimo & Máximo \\
\hline 1 & 40,51 & 4,54 & 32,3 & 51,0 \\
2 & 73,09 & 26,32 & 24,0 & 172,8 \\
Total & 56,80 & 24,93 & 24,0 & 172,8 \\
\hline
\end{tabular}

La repetitividad individual para rendimiento de racimos de fruta fresca (RFF) $\mathrm{kg} /$ planta en ambos grupos $2007 \mathrm{y}$ 2008 es 0,10 y la repetitividad del promedio de cosechas es de 0,87 y 0,82 para los grupos 2007 y 2008, respectivamente (Tabla 5). Así, la selección basada en este promedio propicia exactitud selectiva de 0,93 para el grupo 2007 y de 0,90 para el grupo del 2008. Con estos indicadores es posible realizar la selección de genotipos superiores con alto nivel de precisión (Resende, 1997; Resende, 2002).

\section{Tabla 5}

Componentes de varianza asociados a la producción rendimiento en $\mathrm{kg} / \mathrm{planta} \mathrm{de}$ racimos de fruta fresca (RFF) palma aceitera, con material genético de cinco procedencias, estimados por máxima verosimilitud restringida (REML)

\begin{tabular}{lcc}
\hline Componentes de varianza & Elites & Elites \\
& 2007 & 2008 \\
\hline Varianza Fenotípica permanente & 373,902 & 109,023 \\
Varianza ambiental temporaria & 3365,118 & 981,210 \\
Varianza fenotípica individual & 3739,020 & 1090,233 \\
Repetitividad individual & $0,10 \pm 0,80$ & $0,10 \pm 0,11$ \\
repetitividad de media de cosecha & 0,870 & 0,816 \\
Exactitud selectiva & 0,933 & 0,904 \\
\hline Media general & 141,239 & 70,413 \\
\hline
\end{tabular}

Los resultados revelan un limitado potencial para obtener ganancia genética en esta población, esto debido a que la base genética en evaluación es producto de un mejoramiento genético previo que ha sido realizado en el país de procedencia. Es decir sobre la base de una población mejorada no se espera ganancias genéticas altas en la nueva selección.

\section{Tabla 6}

Selección genética asociados a la producción de frutos de palma aceitera

\begin{tabular}{ccccccc}
\hline Orden & Árbol & $\begin{array}{c}\text { Valor } \\
\text { Fenotípico }\end{array}$ & $\begin{array}{c}\text { Valor } \\
\text { Genético }\end{array}$ & $\begin{array}{c}\text { Ganancia } \\
\text { genética }\end{array}$ & $\begin{array}{c}\text { Promedio } \\
\text { Población } \\
\text { mejorada }\end{array}$ & Ganancia \\
\hline 1 & 4 & 16,65 & 157,89 & 16,65 & 157,89 & 11,79 \\
2 & 13 & 12,51 & 153,75 & 14,58 & 155,82 & 10,32 \\
3 & 25 & 12,28 & 153,52 & 13,81 & 131,62 & 9,78 \\
4 & 3 & 8,50 & 149,74 & 12,49 & 153,72 & 8,84 \\
5 & 5 & 8,47 & 149,71 & 11,68 & 152,92 & 8,27 \\
6 & 54 & 8,43 & 149,67 & 11,14 & 152,38 & 7,89 \\
7 & 111 & 7,19 & 148,42 & 10,58 & 151,82 & 7,49 \\
8 & 105 & 6,73 & 147,96 & 10,09 & 151,33 & 7,15 \\
9 & 42 & 6,54 & 147,78 & 9,70 & 150,94 & 6,87 \\
10 & 11 & 6,32 & 147,56 & 9,36 & 150,60 & 6,63 \\
11 & 48 & 5,92 & 147,16 & 9,05 & 150,29 & 6,41 \\
12 & 120 & 5,76 & 147,00 & 8,78 & 150,01 & 6,21 \\
13 & 22 & 5,49 & 146,73 & 8,52 & 149,76 & 6,03 \\
14 & 7 & 5,17 & 146,41 & 8,28 & 149,52 & 5,86 \\
15 & 99 & 5,11 & 146,35 & 8,07 & 149,31 & 5,72 \\
16 & 129 & 6,20 & 76,61 & 6,20 & 76,81 & 8,81 \\
17 & 127 & 9,94 & 75,36 & 5,57 & 75,99 & 7,91 \\
18 & 138 & 4,72 & 75,13 & 5,29 & 75,10 & 7,51 \\
19 & 126 & 4,70 & 75,11 & 5,14 & 75,55 & 7,30 \\
20 & 123 & 4,18 & 74,59 & 4,95 & 7,03 \\
21 & 125 & 4,12 & 74,53 & 4,81 & 6,83 \\
\hline
\end{tabular}


Tabla 7

Comportamiento productivo de plantas elites seleccionadas según procedencia e hibrido - Perú

\begin{tabular}{ccccc}
\hline $\begin{array}{c}\text { Número } \\
\text { de planta }\end{array}$ & Código de planta & País & Hibrido/Variedad & Rendimiento kg/planta \\
\hline 3 & A1L04P26 & Benin & 2301 & 265,6 \\
4 & A1L08P11 & Benin & 2301 & 375,86 \\
5 & A1L08P22 & Benin & 2301 & 247,2 \\
7 & A1L11P17 & Benin & 2301 & 221,89 \\
11 & A1L15P17 & Benin & 2301 & 213,36 \\
13 & A1L22P21 & Benin & 2301 & 331,2 \\
22 & A1L41P17 & Benin & 2301 & 214,56 \\
25 & A1L59P07 & Benin & 2301 & 243 \\
42 & A2L116P06 & Benin & 2401 & 228 \\
48 & A2L102P11 & Benin & 2401 & 218 \\
54 & A2L83P14 & Benin & 2401 & 210,4 \\
99 & A5L36P18 & Costa de marfil & Cirad & 228 \\
105 & A5L44P24 & Costa de marfil & Cirad & 224,4 \\
111 & A5L64P02 & Costa de marfil & Cirad & 117 \\
120 & A5L73P28 & Costa de marfil & ASD & 115,2 \\
123 & C10L06P25 & Costa rica & ASD & 133,22 \\
125 & C10L06P28 & Costa rica & ASD & 116,48 \\
126 & C10L06P30 & Costa rica & ASD & 137,28 \\
127 & C10L06P27 & Costa rica & ASD & ASD \\
129 & C10L06P24 & Costa rica & & \\
\hline
\end{tabular}

Los resultados de la selección genética, revelan un bajo potencial para obtener ganancia genética en esta población. La selección y clonación de los 15 mejores individuos deberá propiciar una ganancia genética de 5,72\%, elevando la productividad media anual por planta de $141,24 \mathrm{~kg}$ RFF/año para 149,31 kg RFF/año (Tabla 6). Esto es debido al hecho de que la especie demuestra niveles de uniformidad dentro de cada procedencia, esto debido a que las semillas son mejoradas en su país de procedencia y por ello la existencia de variabilidad es baja. Así, la mejora genética de la palma aceitera en nuestro país estaría basada en esta nueva base genética, quedando en el corto plazo la clonación de las plantas elites y ampliar la frontera agrícola.

En las plantas del grupo más joven que va desde el 16 al 21 (Tabla 6), se observa que también la ganancia genética es mínima, es decir si logramos clonar las 6 mejores plantas $(16,17,18,19,20$, y 21), se lograría una ganancia genética al orden de $6,83 \%$ es decir pasar de una media de $70,41 \mathrm{~kg}$ RFF/planta/año a 75,22 kg RFF/planta/año. A este nivel sólo se reportó plantas de la procedencia de Costa Rica y Costa de Marfil.

De acuerdo a la codificación de las plantas elites, se observa que no existe una planta de procedencia Colombiana y de Ecuador y esto confirme la realidad productiva, que son de baja respuesta en nuestras condiciones y por ello han dejado de importar semillas de esta procedencia.

\section{Conclusiones}

Se ha realizado la selección genética, sobre la cual se ha logrado la selección de 21 plantas elites, con ganancias genéticas que van entre $5,72 \%$ a $11,79 \%$.

Las plantas identificadas del grupo 2007, superan los 200 kilos en rendimiento de RFF/año y las plantas del grupo 2008 no han llegado a superar los 200 kilos.

La población en estudio, tiene baja variabilidad genética, atribuyendo mayor variabilidad por el efecto del medio ambiente, esto debido a que el material genético es de procedencia mejorada genéticamente.

Se ha identificado material genético elite de 3 procedencias (Benin, Costa de Marfil 
y Costa Rica), lo mismo que involucran a 4 híbridos 2301, 2401, CIRAD y ASD.

El material genético de Colombia, no ha sobresalido en la selección, es posible que esto confirme la poca introducción del material genético a nuestro país por su bajo rendimiento en nuestras condiciones.

\section{Referencias bibliográficas}

Gobierno Regional de Ucayali. 2011. Situación Actual de la Palma Aceitera en el Perú. Exposición en Power Point.

Oliva, C.; Resende, M.D.V. 2008. Mejoramiento genético y taza de autofecundación del camu camu arbustivo en la amazonía peruana. Rev. Bras. Frutic. (Jaboticabal) 30(2): 450-454.
Oliva, C.; Benito, J.; Acuña, R.; Bocanegra, A.; Baltazar, J. 2014. Estimación de la repetitividad y selección genética de árboles de cacao aromático con material genético de EE-INIA-San Martin y de la UC de Lebuaf, en Perú. Scientia Agropecuaria 5: 59-64.

Pedrozo, C.A.; Benites, F.R.G.; Barbosa, M.H.P.; de Resende, M.D.V.; da Silva, F.L. 2009. Eficiência de Índices de Seleção Utilizando la Metodología Reml/Blup en el Melhoramento de la Cana-DeAçúcar. Scientia Agraria (Curitiba) 10(1): 31-36.

Resende, M.D.V.; Oliveira, E.B. 1997. Sistema "SELEGEN" - Seleção Genética Computadorizada para o Melhoramento de Espécies Perenes. Revista PAB (Colombo) 32(9): 931-939.

Resende, M.D.V. 1997. Software SELEGEN REML/BLUP. EMBRAPA, Colombo. Manual Selegen.

Resende, M.D.V. 2002. Genética biométrica e estatística no melhoramento de plantas perenes. Brasília: Embrapa Informação Tecnológica, 975p. 